PEYTON, B. 1999. Spectacled Bear Conservation Action Plan. Pp. 157-198 En: Bears status survey and conservation plan (Servheen, C., S. Herrero \& B. Peyton eds.), IUCN/SSC Bear and Polar Bear Specialist Groups, Gland, Switzerland and Cambridge, UK.

PROAVES.2013. Regresa el Oso de Anteojos a Anorí, Antioquia. Disponible en: http://www.proaves.org/regresa-el-oso-de-anteojos-a-anori-antioquia/

WILSON, D., \& R.A MITTERMEIER. 2009. Handbook of the Mammals of the world. Vol. I. Carnivores. Page 488 (D. Wlison and R. A. Mittermeier, Eds.). Lynx Edicions, Barcelona.

\section{Registro notable del Perro de Orejas Cortas Atelocynus microtis (Carnivora: Canidae) en el trapecio amazónico, Colombia.}

\section{Stevensson Ayure - Universidad INCCA de Colombia,} Bogotá, Colombia.

José F. González-Maya - ProCAT Colombia \& The Sierra to Sea Institute, Bogotá, Colombia. jfgonzalezmaya@gmail.com

Atelocynus microtis es una de la especies menos conocidas de carnívoros en el mundo, conocido apenas de registros esporádicos a lo largo de su distribución (Michalski 2010, Leite-Pitman \& Williams 2011). Se distribuye entre Colombia y Bolivia y Ecuador, Perú y Brasil, con muy pocos registros y en ventanas de tiempo relativamente grandes (Berta 1986, LeitePitman \& Williams 2011).

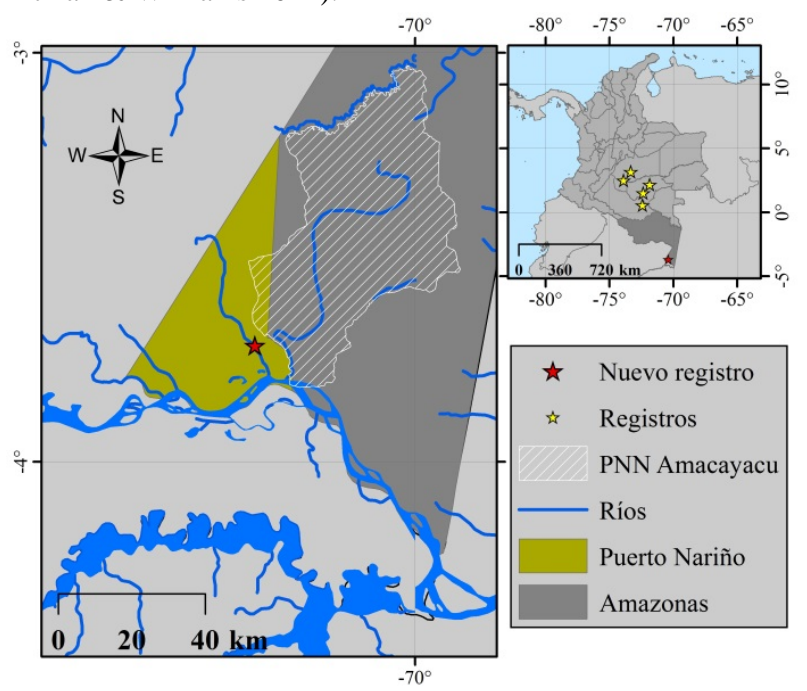

Figura 1. Ubicación de la localidad de los registros de Atelocynus microtis durante el año 2009 en el municipio de Puerto Nariño, Amazonas, Colombia. Estrellas en amarillo en el mapa inserto señalan los registros de colectas.

En Colombia se considera distribuido sólo en la región amazónica, de los 0 a $500 \mathrm{msnm}$ y en los departamentos de Amazonas, Caquetá, Meta y Vaupés (Alberico et al. 2000, Solari et al. 2013). Entre los primeros registros para el país se citan tres especímenes provenientes de las cercanías de Mitú, Vaupés (colectado en 1956), una hembra viva proveniente del río Ariari, Meta y un individuo de Restrepo, Meta; todos identificados por Hernández-Camacho J. y publicados por Hershkovitz (1961). A partir de estos registros y otra información recopilada por Hershkovitz (1961), se considera que la especie se distribuye en el país en la zona amazónica hasta la parte alta del río Orinoco, incluyendo Venezuela, sin embargo, no hay registros que respalden esta afirmación (LeitePitman \& Williams 2011). Adicionalmente, basados en una búsqueda en el GBIF (Global Biodiversity Information Facility 2014) se encontraron 10 registros en colecciones, ocho en la colección de mamíferos del Instituto de Investigaciones Alexander von Humboldt (IAvH) y dos en el Instituto de Ciencias Naturales de la Universidad Nacional de Colombia (ICN); de estos, ninguno contaba con coordenadas exactas y sólo cinco fue posible ubicarlas con los datos de localidad (Figura 1). De estos registros, dos son referenciados para el departamento del Meta, mientras se encontró un registro para los departamentos de Putumayo, Caquetá y Guaviare. En años recientes el uso de cámaras-trampa ha permitido la detección de especies raras y elusivas en zonas previamente desconocidas para muchas de estas (O'Connell et al. 2011).

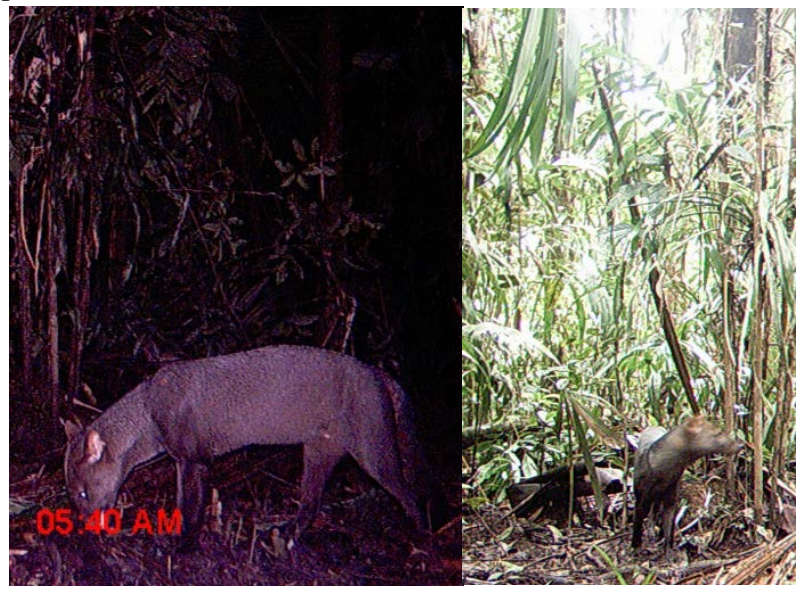

Figura 2. Fotos registradas de Atelocynus microtis durante el año 2009 en el trapecio amazónico, Colombia.

En esta nota presentamos dos registros nuevos de Atelocynus microtis para el municipio de Puerto Nariño, departamento del Amazonas, Colombia. El registro fue obtenido durante un muestreo sistemático de cámaras trampa para evaluar la influencia de la cacería sobre el ensamblaje de mamíferos, en la comunidad Ticuna de San José de Villa Andrea, sobre la ribera del río Loretoyacu, parte del resguardo indígena Aticoya. El muestreo incluyó 12 estaciones dobles de cámaras-trampa, con un esfuerzo de muestreo total de 264 noches-trampa, detectando un total de 16 especies. Los dos registros de Atelocynus microtis se obtuvieron en una misma estación, en la zona más alejada del poblado dentro del estudio ( $3^{\circ} 17^{\prime} 4,84^{\prime \prime} \mathrm{S}$, $70^{\circ} 23^{\prime} 31,33$ 'W). El primer registro se obtuvo el 4 de junio a las $10 \mathrm{~h} 00$ y el segundo registro fue obtenido el 18 de junio a las 5h40 ambas en el año 2009 (Figura 2). 
Este nuevo registro, a pesar de estar dentro del área esperada de distribución de la especie, es un registro notable al aportar nueva información para la especie en el país. Tratándose de unos de los carnívoros más raros del mundo, la información sobre registros de ocurrencia es fundamental para evaluar su estado de conservación, y en específico para esta especie es necesario realizar más estudios ecológicos y de conservación que permitan un adecuado manejo y planificación para la preservación de la especie. Agradecemos a la Fundación Omacha y el Fondo para Especies Amenazadas por el financiamiento parcial de esta investigación.

\section{Referencias}

ALBERICO, M., A. et al. 2000. Mamíferos (Synapsida: Theria) de Colombia. Biota Colombiana 1:43-75.

BERTA, A. 1986. Atelocynus microtis. Mammalian Species 256:1-3.

GLOBAL BIODIVERSITY INFORMATION FACILITY. 2014. GBIF Database of Occurrences. (Facility GBI ed.), Copenhagen, Denmark.

HERSHKOVITZ, P. 1961. On the South American small-eared zorro Atelocynus microtis Sclater (Canidae). Fieldiana Zoology 39:505-523.

LEITE-PITMAN, M. R. P. AND R. S. R. WILLIAMS. 2011. Atelocynus microtis. http://www.iucnredlist.org. Accessed 13 April 20142014.

MICHALSKI, F. 2010. The bush dog Speothos venaticus and short-eared dog Atelocynus microtis in a fragmented landscape in southern Amazonia. Oryx 44:300-300.

O'CONNELL, A. F., et al. 2011. Camera Traps in Animal Ecology: Methods and Analyses. Springer, New York, USA 286.

SOLARI, S., et al. 2013. Riqueza, endemismo y conservación de los mamíferos de Colombia. Mastozoología Neotropical 20:301-365.

Primer Reporte de leucismo en Artibeus lituratus (Olfers, 1818) (Phyllostomidae: Stenodermatinae) en el Caribe colombiano.

Gina Olarte-González - Universidad El Bosque \& Proyecto de Conservación de Aguas y Tierras, ProCAT Colombia, Bogotá, Colombia.giolgon@gmail.com.

I. Mauricio Vela-Vargas - Proyecto de Conservación de Aguas y Tierras, ProCAT Colombia, Bogotá, Colombia.

\section{Plinio Camargo}

Tellis Jhonson

Álvaro Jiménez - Parque Nacional Natural Tayrona, Santa Marta, Colombia..

El leucismo es una hipo-pigmentación del tegumento en el que el individuo presenta parches irregulares o incluso la totalidad del cuerpo despigmentado, pero mantiene la coloración normal de los ojos y garras, lo cual puede ser descrito erróneamente como albinismo parcial (Roncancio \& Ramírez-Chaves 2008, Marín-Vásquez et al. 2010, Tabea-Treitler et al. 2013 \& Velandia-Perilla et al. 2013). Las causas que explican el leucismo se han explicado cómo la expresión de alelos mutantes o anomalías en la diferenciación embrionaria de células pigmentarias (Brack et al. 2005). Para el caso puntual de la especie Artibeus. lituratus (Olfers, 1818) se ha reportado en México (García-Morales et al. 2013, López-Wilchis \& León Galván 2012), Brasil (Souza et al. 2013) y para Colombia en el departamento de Valle del Cauca (Velandia-Perilla et al. 2014). A nivel de género también se ha reportado está condición en Artibeus jamaicencis en la isla Santa Catalina del Archipiélago de San Andrés (Marín-Vásquez et al. 2010). Está registrado en la literatura que dentro del género Artibeus se presenta predisposición para estas aberraciones de color (HérnandézMinjagos 2009).

El individuo fue capturado en mayo de 2012 en las proximidades de la Bahía de Cinto a 20 metros de altitud, en una zona de bosque húmedo tropical (11²19'29,3' $\mathrm{N}$, 73313,0”W) dentro del Parque Nacional Natural Tayrona, Magdalena, Colombia; a través del uso de redes de niebla de 9x3 m. a nivel de sotobosque. Artibeus lituratus se caracteriza por la presencia de molares $2 / 3$, con una coloración café no jaspeada y líneas faciales prominentes (Gardner 2008). El individuo fue un macho adulto cuyas medidas morfométricas fueron: LC: 73,30 mm, LP: 13,76 mm, Calcaneo: 5,38 mm, LO: 16,24 mm, LA: 58,06 mm, HN: 13,66 mm y 58 gramos de peso (Figura. 1). Esta investigación se desarrolló dentro del proyecto "Monitoreo y creación de capacidades para la protección y manejo del Parque Nacional Natural Tayrona: Enfoque en mamíferos como herramientas de planificación”, llevado a cabo por ProCAT Colombia con la asistencia técnica de Parques Nacionales Naturales de Colombia.

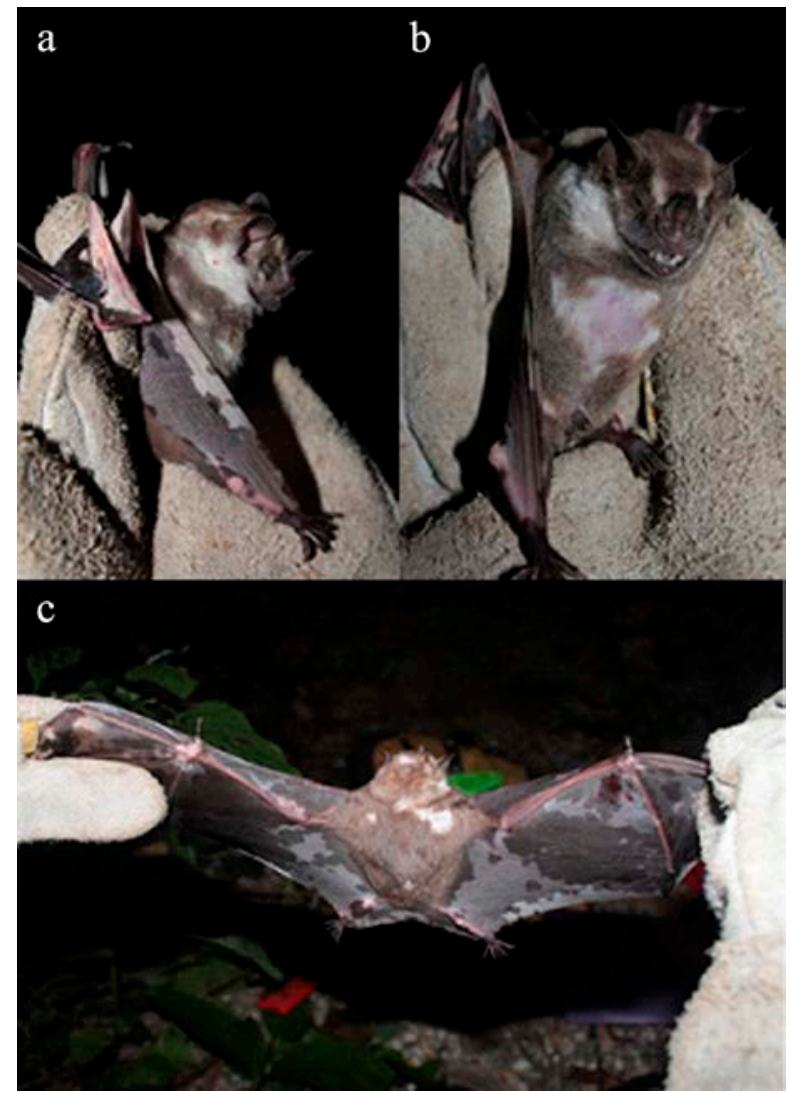

Figura 1. Individuo de A. lituratus con leucismo. a) Vista lateral, b) Vista ventral y c) Vista dorsal 